\title{
Nuclear polarization in heavy atoms and superheavy quasiatoms
}

\author{
Günter Plunien \\ Department of Physics and Astronomy, Vanderbilt University, Nashville, Tennessee 37235 \\ Berndt Müller* and Walter Greiner \\ Institut für Theoretische Physik der Universität Frankfurt, Robert-Mayer-Strasse 8-10, Postfach 111 932, \\ D-6000 Frankfurt am Main, Federal Republic of Germany \\ Gerhard Soff \\ Gesellschaft für Schwerionenforschung, Planckstrasse 1, Postfach 110 552, D-6100 Darmstadt, Federal Republic of Germany
}

(Received 23 July 1990)

\begin{abstract}
We consider the contribution of nuclear polarization to the Lamb shift of $K$ - and $L$-shell electrons in heavy atoms and quasiatoms. Our formal approach is based on the concept of effective photon propagators with nuclear-polarization insertions treating effects of nuclear polarization on the same footing as usual QED radiative corrections. We explicitly derive the modification of the photon propagator for various collective nuclear excitations and calculate the corresponding effective self-energy shift perturbatively. The energy shift of the $1 s_{1 / 2}$ state in ${ }_{92}^{238} \mathrm{U}$ due to virtual excitation of nuclear rotational states is shown to be a considerable correction for atomic high-precision experiments. In contrast to this, nuclear-polarization effects are of minor importance for Lamb-shift studies in ${ }_{82}^{208} \mathrm{~Pb}$.
\end{abstract}

\section{INTRODUCTION}

Future experimental abilities to prepare highly ionized atoms and possibly even bare heavy nuclei up to uranium will open a new generation of high-precision experiments. One of the fascinating aspects is that studies with almost bare nuclei may provide new sensitive tests of quantum electrodynamics in strong external electromagnetic fields. For the analysis of proposed precision experiments with hydrogen-, helium- or lithiumlike uranium a precise knowledge of the electronic spectrum is required. In heavy atoms several effects give rise to measurable contributions to the total binding energy of atomic states. Of those the effect of finite nuclear size as well as quantum electrodynamical (QED) radiative corrections such as vacuum polarization and self-energy effects are well known. ${ }^{1-3}$

At very high precision the additional energy shift of $K$-shell electrons due to nuclear polarization may become relevant. The interactions between leptons and nuclear degrees of freedom have been extensively studied in muonic atoms ${ }^{4-6}$ (see, e.g., Ref. 6 and references therein). In muonic atoms the resulting energy corrections can become relatively large since binding energies of muonic states are comparable in magnitude with typical nuclear excitation energies. Analogous calculations performed in the case of electronic atoms ${ }^{7,8}$ show that the corresponding energy shifts are much smaller. One reason for that is the much larger size of the electronic orbits. However, the influence of this contribution increases when the interaction with low-lying nuclear rotational modes is taken into account. For heavy nuclei, such as uranium, the electronic transition energies are comparable in magnitude with the nuclear excitation energies.

It is useful to set up a more general and rigorous treatment of nuclear polarization within the framework of QED, where these contributions appear on the same footing as the QED-radiative corrections. Our treatment of the energy shift is based on the concept of dressed photon propagators containing nuclear-polarization insertions. Effects due to nuclear polarization thus appear as part of the radiative corrections to electronic levels. We then discuss the treatment of nuclear excitations laying particular emphasis on collective nuclear modes. We will derive effective propagators for nuclear surface vibrations, rotations, and giant resonances, respectively.

In order to get an insight into how the energy correction increases for large values of the nuclear charge $Z$, we performed model calculations for superheavy quasiatoms formed in heavy-ion collisions. A static calculation for a system with a united charge $Z=170$ reveals at least qualitative features of the $Z$-dependence and gives a rough estimate for the order of magnitude. As a function of $Z$ the binding energy of atomic levels rises monotonically with increasing nuclear charge. The binding energy of a quasiatomic $1 s$ state is supposed to exceed twice the electron rest energy for $Z_{\mathrm{cr}} \approx 172 .^{9,10}$ Since QEDvacuum polarization and self-energy contributions to the binding energy almost cancel ${ }^{11,12}$ one would like to know to what extent nuclear-polarization effects may alter the value for the critical charge $Z_{\mathrm{cr}}$.

This paper is organized as follows: In Secs. II A and II B we review some theoretical formalism useful for our 
purposes here. The effective self-energy correction in first-order perturbation theory will be evaluated in Sec. II C. In Sec. III we present some results of our calculations. In Sec. III A we point out some numerical aspects to illustrate the basic difficulties one is faced with when performing such calculations in the case of light leptons. We then discuss our result for the contribution to the $1 s$ Lamb shift in $\mathrm{Pb}$ and $\mathrm{U}$ presented earlier. ${ }^{13,14}$ Finally in Sec. III C, we turn to the model calculation of the energy shift for a system with charge $Z=170$ to estimate the effect in superheavy quasiatoms. We will use units with $\hbar=c=1, \alpha=\frac{e^{2}}{4 \pi}$.

\section{THEORETICAL APPROACH}

We start with a description of the formalism used here in which the effects of nuclear polarization on the atomic level spectrum naturally appear as effective radiative corrections. The general concepts of nuclear-polarization insertions and dressed interactions allow a systematic treatment of nuclear-polarization corrections to an arbitrary QED process. Effective photon propagators will be specified for collective nuclear excitations and in particular the effective self-energy correction will be evaluated.

\section{A. The interaction operator, nuclear-polarization} insertion, and effective photon propagator

Although the interaction between electrons and the nucleus is in principle known, a complete quantum field theoretical treatment of the atomic bound-state problem has its practical limitations. Nevertheless the major corrections to the energy of atomic states can be obtained by means of perturbation theory. In the case of highly ionized atoms or even bare nuclei a good approximation is achieved by considering the following scenario: The nuclear charge distribution is described by the electromagnetic current density

$$
\hat{j}_{\text {nuc }}^{\mu}(x)=j_{\text {ext }}^{\mu}(\mathbf{x})+\hat{j}_{\text {fluc }}^{\mu}(x) .
$$

It consists of a static equilibrium (c-number) part $j_{\text {ext }}^{\mu}$ (only an external charge density $\rho_{\text {ext }}$ in the rest frame of the nucleus) characterizing the nucleus in its ground state and a second quantized, time-dependent part $\hat{j}_{\text {fluc }}^{\mu}$ characterizing intrinsic dynamics of the nuclear charge density due to external electromagnetic excitations. The Dirac current

$$
\hat{j}_{e}^{\mu}(x)=-\frac{e}{2}\left[\hat{\bar{\Psi}}(x), \gamma^{\mu} \hat{\Psi}(x)\right]
$$

interacts with the electromagnetic field

$$
\hat{A}^{\mu}(x)=A_{\mathrm{ext}}^{\mu}(\mathbf{x})+\hat{A}_{\mathrm{rad}}^{\mu}(x)+\hat{A}_{e}^{\mu}(x),
$$

where the classical external field $A_{\text {ext }}^{\mu}$ is created by the classical static nuclear source $j_{\text {ext }}^{\mu}$. We introduce a total radiation field $\hat{A}_{\text {rad }}^{\mu}$ as the sum

$$
\hat{A}_{\text {rad }}^{\mu}(x)=\hat{A}_{\text {free }}^{\mu}(x)+\hat{A}_{\text {fluc }}^{\mu}(x)
$$

of the free photon field $\hat{A}_{\text {free }}^{\mu}$ and the fluctuating part $\hat{A}_{\text {fluc }}^{\mu}$ generated by the nuclear transition current $\hat{j}_{\text {fluc }}^{\mu}$. Since we intend to consider one- or few-electron systems the electron-electron interaction mediated by the part $\hat{A}_{e}^{\mu}$ will be neglected.

The interaction between the electron-positron field and internal nuclear degrees of freedom is described by the interaction Hamiltonian

$$
\hat{H}_{\mathrm{int}}(t)=\hat{H}^{\mathrm{rad}}(t)+\hat{H}^{\delta m}(t),
$$

with

$$
\begin{aligned}
\hat{H}^{\mathrm{rad}} & =\int d^{3} x \hat{j}_{e}^{\mu}(x) \hat{A}_{\mu}^{\mathrm{rad}}(x), \\
\hat{H}^{\delta m} & =\frac{1}{2} \delta m \int d^{3} x[\hat{\bar{\Psi}}(x), \hat{\Psi}(x)] .
\end{aligned}
$$

The first term $\hat{H}^{\text {rad }}$ describes the interaction between the Dirac field and the total radiation field (4) while the term $\hat{H}^{\delta m}$ has to be added for reasons of mass renormalization. Accordingly the mass correction $\delta m$ to the (unphysical) bare mass of the noninteracting electron is determined such that the observable mass of the interacting (physical) electron becomes $m=1$.

It is most adequate for the problem set up here to work in the Furry picture (bound state interaction picture) (Ref. 15) where the external field problem is already solved. Accordingly one expands the field operators $\hat{\Psi}$ in terms of the one particle basis

$$
\hat{\Psi}(x)=\sum_{i(>F)} \hat{b}_{i} e^{-\varepsilon_{i} t} \psi_{i}(\mathbf{x})+\sum_{i(<F)} \hat{d}_{i}^{\dagger} e^{-\varepsilon_{i} t} \psi_{i}(\mathbf{x})
$$

where the $\psi_{i}$ obey the Dirac equation

$$
\begin{array}{r}
\left\{i \gamma^{0} \gamma \cdot\left[\nabla+i e \mathbf{A}_{\mathrm{ext}}(\mathbf{x})\right]+e A_{\mathrm{ext}}^{0}(\mathbf{x})+\gamma^{0} m\right\} \psi_{i}(\mathbf{x}) \\
=\varepsilon_{i} \psi_{i}(\mathbf{x}),
\end{array}
$$

and thus taking into account the interaction between electron and the electromagnetic field of the static nucleus up to all orders in $Z \alpha$. The time evolution of a (normalized) one-particle state $\left|\Phi_{i}(t)\right\rangle$ is given via the evolution operator $\hat{U}\left(t, t^{\prime}\right)$ according to

$$
\begin{aligned}
\left|\Phi_{i}(t)\right\rangle & =\hat{U}\left(t, t^{\prime}\right)\left|\Phi_{i}\left(t^{\prime}\right)\right\rangle \\
& =T \exp \left(-i \int_{t^{\prime}}^{t} d \tau \hat{H}_{\mathrm{int}}(\tau)\right)\left|\Phi_{i}\left(t^{\prime}\right)\right\rangle .
\end{aligned}
$$

This formal equation should also imply adiabatic switching off of the interaction $\hat{H}_{\text {int }}$ at $t \rightarrow \pm \infty$ and that $\left|\Phi_{i}\right\rangle$ asymptotically becomes again an eigenstate of $\hat{H}_{0}$, i.e., $\left|\Phi_{i}(t \rightarrow \pm \infty)\right\rangle=\hat{a}_{i}^{\dagger}|0\rangle \equiv\left|\Phi_{i}^{0}\right\rangle$. In accordance with Gell-Mann and Low's theorem ${ }^{16}$ the energy shift of a given electron bound state $\left|\Phi_{i}^{0}\right\rangle=|i\rangle$ due to the interaction with the total radiation field is expressed by the Tomonaga-Schwinger equation ${ }^{17}$ 


$$
e^{-i \Delta E_{i} T}=\left\langle\Phi_{i}^{0}\left|\mathrm{~T} \exp \left(-i \int_{-T / 2}^{T / 2} d \tau \hat{H}^{\mathrm{int}}(\tau)\right)\right| \Phi_{i}^{0}\right\rangle
$$

in the limit of large time differences $T$. The energy shift given by (9) is in general complex valued. While the real part describes the physical energy shift of the bound state, the imaginary part gives the decay rate of the bound state due to possible transitions of the electron into lower-lying unoccupied bound states. Thus only the energy correction for the $1 s$ state is real.

Equation (9) contains the complete information about the change of the electron spectrum due to the interaction with the free radiation field $\hat{A}_{\text {free }}^{\mu}$ and with the fluctuations $\hat{A}_{\text {fluc }}^{\mu}$ arising from nuclear transitions. When performing perturbation expansion of (9) one is naturally led to Feynman diagrams with dressed (inner) photon lines representing an effective photon propagator defined by the time-ordered product of the total radiation field operators

$$
i \mathcal{D}_{\mu \nu}\left(x, x^{\prime}\right)=\left\langle 0\left|T\left[\hat{A}_{\mu}^{\mathrm{rad}}(x) \hat{A}_{\nu}^{\mathrm{rad}}\left(x^{\prime}\right)\right]\right| 0\right\rangle .
$$

The "vacuum" expectation value implies that the nucleus is considered to be in its ground state. We can rewrite the definition (10) as the sum of the free photon propagator $i D_{\mu \nu}\left(x-x^{\prime}\right)=\left\langle 0\left|T\left[\hat{A}_{\mu}^{\text {free }}(x) \hat{A}_{\nu}^{\text {free }}\left(x^{\prime}\right)\right]\right| 0\right\rangle$ and a polarization correction

$$
\begin{aligned}
& \tilde{\mathcal{D}}_{\mu \nu}\left(x, x^{\prime}\right) \\
& \quad=\int d^{4} x_{1} d^{4} x_{2} D_{\mu \alpha}\left(x-x_{1}\right) \Pi^{\alpha \beta}\left(x_{1}, x_{2}\right) D_{\beta \nu}\left(x_{2}-x^{\prime}\right),
\end{aligned}
$$

which defines the reducible polarization insertion $\Pi^{\alpha \beta}$. The polarization tensor can be expressed as current correlation function

$$
i \Pi^{\alpha \beta}\left(x_{1}, x_{2}\right)=\left\langle 0\left|T\left[\hat{j}_{\text {fluc }}^{\alpha}\left(x_{1}\right) \hat{j}_{\text {fluc }}^{\beta}\left(x_{2}\right)\right]\right| 0\right\rangle .
$$

It contains all nuclear degrees of freedom which can be virtually excited by means of virtual photon exchange between a bound electron and the nucleus. Equation (12) is only exact if $\hat{j}_{\text {fluc }}^{\alpha}$ represents the exact Heisenberg operator. Since the exact transition current is not known from first principles Eq. (12) can only be specified by applying particular nuclear models. Consequently the effective interaction (11) becomes model dependent. Before we turn to its explicit derivation let us give the result for the energy shift due to nuclear polarization in lowest-order perturbation theory. Expanding (9) in lowest nonvanishing order in $\hat{H}^{\text {rad }}$ reveals that nuclear-polarization contributions now appear as part of the QED radiative corrections. Applying Wick's theorem ${ }^{18}$ and arranging terms depending on whether they contain free photon lines $D_{\mu \nu}$ or effective photon lines $\tilde{\mathcal{D}}_{\mu \nu}$ leads to the energy shift

$$
\begin{aligned}
\Delta E_{i}^{\mathrm{NP}}= & i \alpha \int d^{3} x_{1} d^{3} x_{2} \bar{\psi}_{i}\left(\mathbf{x}_{1}\right) \gamma^{\mu} \int d E S_{F}\left(\mathbf{x}_{1}, \mathbf{x}_{2}, E\right) \tilde{\mathcal{D}}_{\mu \nu}\left(\mathbf{x}_{1}, \mathbf{x}_{2}, E-E_{i}\right) \gamma^{\nu} \psi_{i}\left(\mathbf{x}_{2}\right) \\
& -2 i \alpha \int d^{3} x_{1} d^{3} x_{2} j_{i}^{\mu}\left(\mathbf{x}_{1}\right) \tilde{\mathcal{D}}_{\mu \nu}\left(\mathbf{x}_{1}, \mathbf{x}_{2}, E=0\right) \operatorname{Tr}\left[\gamma^{\nu} S_{F}\left(\mathbf{x}_{1}, \mathbf{x}_{2}, E=0\right)\right]
\end{aligned}
$$

where the Fourier-transformed electron propagator $S_{F}$ satisfies the equation

$$
\left(E-\left\{i \gamma^{0} \boldsymbol{\gamma} \cdot\left[\boldsymbol{\nabla}+i e \mathbf{A}_{\mathrm{ext}}(\mathbf{x})\right]+e A_{\mathrm{ext}}^{0}(\mathbf{x})+\gamma^{0} m\right\}\right) S_{F}\left(\mathbf{x}, \mathbf{x}^{\prime}, E\right)=\gamma^{0} \delta\left(\mathbf{x}-\mathbf{x}^{\prime}\right)
$$

The first term in Eq. (13) represents an effective selfenergy diagram. As we will see below it contains the contribution calculated earlier ${ }^{7,8}$ by means of second-order (Schrödinger) perturbation theory. The second term describes a contribution due to the interaction between the induced vacuum polarization and nuclear degrees of freedom. In the calculation presented here we will focus on the effective self-energy.

When calculating $\tilde{\mathcal{D}}_{\mu \nu}$ we will neglect possible distortions of the nuclear excitation spectrum due to the presence of the electron. Such effects, which occur in the case of muonic atoms, are expected to be of minor importance here. Accordingly the time evolution of the nuclear transition current $\hat{j}_{\text {fluc }}^{\mu}$ is governed by the nuclear (model) Hamiltonian $\hat{H}_{\text {nuc }}$. For our purposes we will further neglect the contribution of the vector current $\hat{\mathbf{j}}$ fluc because the velocities associated with intrinsic nuclear dynamics are mainly nonrelativistic. Keeping the $\Pi^{00}$ component of the polarization tensor we thus deal only with the longitudinal part $\tilde{\mathcal{D}}_{00}$ of the effective propagator. We will calculate the part

$$
\Delta E_{i}=i \alpha \int d^{3} x_{1} d^{3} x_{2} \psi_{i}^{+}\left(\mathbf{x}_{1}\right) \int d E S_{F}\left(\mathbf{x}_{1}, \mathbf{x}_{2}, E_{i}-E\right) \tilde{\mathcal{D}}_{00}\left(\mathbf{x}_{1}, \mathbf{x}_{2}, E\right) \gamma^{0} \psi_{i}\left(\vec{x}_{2}\right)
$$


of the effective self-energy.

\section{B. Polarization insertions and effective photon propagators for collective nuclear excitations}

Having set up the more general framework we now derive explicit expressions for the nuclear-polarization insertion and for the modification of the effective propagator. We will give some details for the cases of the harmonic surface vibrator and giant resonances.

To begin, let us recall the well-known parametrization of the nuclear surface

$$
R(\Omega, t)=R_{0}\left(1+\sum_{L, M} \alpha_{L M}^{*} Y_{L M}(\Omega)\right)
$$

where $R_{0}$ denotes the radius of a homogeneously charged sphere characterizing the nucleus in its ground state. In the harmonic approximation the collective dynamics of the nuclear surface is described by the collective Hamiltonian $\hat{H}_{c}$ (Refs. 19 and 20)

$$
\hat{H}_{c}=\frac{1}{2} \sum_{L, M} \frac{1}{B_{L}}\left|\hat{\pi}_{L M}\right|^{2}+\frac{1}{2} \sum_{L, M} C_{L}\left|\hat{\alpha}_{L M}\right|^{2}
$$

where $\hat{\pi}_{L M}=B_{L} \hat{\dot{\alpha}}_{L M}^{\dagger}$ are the conjugate momenta to the collective coordinates $\hat{\alpha}_{L M} . B_{L}$ and $C_{L}$ stand for the inertia and stiffness parameters, respectively. The dynamical evolution of the Heisenberg operators $\hat{\alpha}_{L M}$ is given by

$$
\begin{aligned}
& \hat{\alpha}_{L M}(t)=e^{i \hat{H}_{c} t} \hat{\alpha}_{L M}(0) e^{-i \hat{H}_{c} t}, \\
& \hat{\dot{\alpha}}_{L M}(t)=i\left[\hat{H}_{c}, \hat{\alpha}_{L M}(t)\right],
\end{aligned}
$$

which implies the time dependence

$$
\hat{\alpha}_{L M}(t)=\hat{\alpha}_{L M}(0) e^{-i \omega_{L} t}
$$

for the free shape oscillations. The operators $\hat{\alpha}_{L M}$ and $\hat{\pi}_{L M}$ are related to phonon creation (annihilation) operators $\hat{\beta}_{L M}^{\dagger}\left(\hat{\beta}_{L M}\right)$ via a canonical transformation

$$
\hat{\beta}_{L M}^{\dagger}=\left(\frac{\omega_{L} B_{L}}{2}\right)^{\frac{1}{2}}\left(\hat{\alpha}_{L M}-\frac{i}{\omega_{L} B_{L}} \hat{\pi}_{L M}^{\dagger}\right)
$$

leading to the form

$$
\hat{H}_{c}=\sum_{L, M} \omega_{L}\left(\hat{\beta}_{L M}^{\dagger} \hat{\beta}_{L M}+\frac{1}{2}\right)=: \hat{H}_{c}:+E_{0}
$$

for the Hamiltonian.

By means of the surface parametrization $R(\Omega, t)$ and requiring that the charge is always homogeneously distributed, i.e., with an average density $\rho_{0}=\frac{3 Z e}{4 \pi R_{0}^{3}}$, then, as a consequence of volume conservation, the following expression for the charge density results:

$$
\begin{aligned}
& \rho(\mathbf{r}, t)=\rho_{\text {ext }}(\mathbf{r})+\rho_{\text {fluc }}(\mathbf{r}, t)=\rho_{0} \Theta(R-r), \\
& \rho_{\text {ext }}(\mathbf{r})=\rho_{0} \Theta\left(R_{0}-r\right),
\end{aligned}
$$

$$
\begin{aligned}
\rho_{\text {fluc }}(\mathbf{r}, t)=\rho_{0} \delta\left(R_{0}-r\right) \sum_{n=1}^{3} & \left.\frac{R_{0}^{n}}{n !}\left(\frac{d^{n}}{d R^{n}} \Theta(R-r)\right)\right|_{R=R_{0}} \\
& \times[\epsilon(\Omega, t)]^{n}
\end{aligned}
$$

together with the abbreviation $\epsilon(\Omega, t)=[1+$ $\left.\sum_{L M} \alpha_{L M}^{*}(t) Y_{L M}(\Omega)\right]$. The corresponding Hermitian transition charge-density operator up to lowest order in $\hat{\alpha}_{L M}$ reads

$$
\begin{array}{r}
\hat{\rho}_{\text {fluc }}(\mathbf{r}, t)=\rho_{0} \delta\left(R_{0}-r\right) \sum_{L, M} \hat{\alpha}_{L M}^{\dagger}(t) Y_{L M}(\Omega)+O\left(\hat{\alpha}^{2}\right) \\
=R^{-(L+2)} \delta\left(R_{0}-r\right) \sum_{L, M} \hat{Q}_{L M}(t) Y_{L M}(\Omega), \\
L \geq 2 \quad(21)
\end{array}
$$

where the electric multipole operator $\hat{Q}_{L M}$ defined as

$$
\hat{Q}_{L M}(t)=\int d^{3} r \hat{\rho}_{\text {fluc }}(\mathbf{r}, t) r^{L} Y_{L M}(\Omega)
$$

has been introduced. We should note here that assuming irrotational flow an expression for the nuclear vector current $\hat{\mathbf{j}}_{\text {fluc }}$ can easily be derived. However, we will neglect these contributions to the polarization because they are of the order $O\left(v_{\text {coll }} / c\right)$ and thus of minor importance. Accordingly we only need to calculate the $\Pi^{00}$ component of the corresponding nuclear-polarization tensor. The density correlation function $\Pi^{00}$ can be calculated applying the definition (12). Since we assume that the density fluctuation is time-evolved by the collective Hamiltonian (20) $\Pi^{00}$ becomes homogeneous in time

$$
\begin{aligned}
i \Pi^{00}\left(\mathbf{r}, \mathbf{r}^{\prime}, t-t^{\prime}\right) & =\left\langle 0\left|T\left[\hat{\rho}_{\text {fluc }}(\mathbf{r}, t) \hat{\rho}_{\text {fluc }}\left(\mathbf{r}^{\prime}, t^{\prime}\right)\right]\right| 0\right\rangle \\
& =\Theta\left(t-t^{\prime}\right) \sum_{\nu}\left\langle 0\left|\hat{\rho}_{\text {fluc }}(\mathbf{r}, 0) e^{-i: \hat{H}_{c}:\left(t-t^{\prime}\right)}\right| \nu\right\rangle\left\langle\nu\left|\hat{\rho}_{\text {fluc }}\left(\mathbf{r}^{\prime}, 0\right)\right| 0\right\rangle \\
& +\Theta\left(t^{\prime}-t\right) \sum_{\nu}\left\langle 0\left|\hat{\rho}_{\text {fluc }}\left(\mathbf{r}^{\prime}, 0\right) e^{-i: \hat{H}_{c}:\left(t-t^{\prime}\right)}\right| \nu\right\rangle\left\langle\nu\left|\hat{\rho}_{\text {fluc }}(\mathbf{r}, 0)\right| 0\right\rangle,
\end{aligned}
$$

which gives after temporal Fourier transformation

$$
\Pi^{00}\left(\mathbf{r}, \mathbf{r}^{\prime}, \omega\right)=\sum_{\nu}\left\langle 0\left|\left(\frac{\hat{\rho}_{\text {fluc }}(\mathbf{r}, 0)}{\omega-: \hat{H}_{c}:+i \eta}-\frac{\hat{\rho}_{\text {fluc }}\left(\mathbf{r}^{\prime}, 0\right)}{\omega+: \hat{H}_{c}:-i \eta}\right)\right| \nu\right\rangle\left\langle\nu\left|\hat{\rho}_{\text {fluc }}\left(\mathbf{r}^{\prime}, 0\right)\right| 0\right\rangle .
$$


The vacuum $|0\rangle$ represents the ground state of the nucleus defined as $\hat{\beta}_{L M}|0\rangle=0$ and a complete set of one-phonon states $|\nu\rangle=\hat{\beta}_{L M}^{\dagger}|0\rangle=|L M\rangle$ has been inserted for evaluating the matrix elements occurring in the case considered here. In view of (21) we can further write

$$
\begin{gathered}
\Pi^{00}\left(\mathbf{r}, \mathbf{r}^{\prime}, \omega\right)=R_{0}^{-4} \delta\left(R_{0}-r\right) \delta\left(R_{0}-r^{\prime}\right) \sum_{L, M ; L^{\prime}, M^{\prime} ; L^{\prime \prime}, M^{\prime \prime}} \\
R_{0}^{-\left(L+L^{\prime}\right)}\left\langle 0\left|\hat{Q}_{L M}\right| L^{\prime \prime} M^{\prime \prime}\right\rangle\left\langle L^{\prime \prime} M^{\prime \prime}\left|\hat{Q}_{L^{\prime} M^{\prime}}^{\dagger}\right| 0\right\rangle \\
\times \frac{2 \omega_{L^{\prime \prime}}}{\omega^{2}-\omega_{L^{\prime \prime}}^{2}+i \eta} Y_{L M}(\Omega) Y_{L^{\prime} M^{\prime}}^{*}\left(\Omega^{\prime}\right)
\end{gathered}
$$

Using the Wigner-Eckart theorem for evaluating the matrix elements

$$
\left\langle 0\left|\hat{Q}_{L M}\right| L^{\prime \prime} M^{\prime \prime}\right\rangle\left\langle L^{\prime \prime} M^{\prime \prime}\left|\hat{Q}_{L^{\prime} M^{\prime}}^{\dagger}\right| 0\right\rangle=\delta_{L L^{\prime \prime}} \delta_{L L^{\prime}} \delta_{M M^{\prime \prime}} \delta_{M M^{\prime}}\left\langle 0\left\|\hat{Q}_{L}\right\| L^{\prime \prime}\right\rangle\left\langle L^{\prime \prime}\left\|\hat{Q}_{L}^{\dagger}\right\| 0\right\rangle,
$$

and introducing the reduced transition probabilities $B(E L ; L \rightarrow 0)$ for electric transitions from excited states $|L M\rangle=$ $\hat{\beta}_{L M}^{\dagger}|0\rangle$ of the nucleus into its ground state $|0\rangle$, defined as

$$
B(E L ; L \rightarrow 0)=\frac{\left|\left\langle 0\left\|\hat{Q}_{L}\right\| L M\right\rangle\right|^{2}}{2 L+1}
$$

then the polarization function takes the form

$$
\Pi^{00}\left(\mathbf{r}, \mathbf{r}^{\prime}, \omega\right)=\delta\left(R_{0}-r\right) \delta\left(R_{0}-r^{\prime}\right) \sum_{L, M} R_{0}^{-2(L+1)} B(E L ; L \rightarrow 0) \frac{2 \omega_{L}}{\omega^{2}-\omega_{L}^{2}+i \eta} Y_{L M}(\Omega) Y_{L M}^{*}\left(\Omega^{\prime}\right)
$$

Having derived the expression for the polarization insertion we are now in the position to calculate the longitudinal component $\tilde{\mathcal{D}}_{00}$ of the modification of the photon propagator. This is most easily achieved in the Coulomb gauge according to

$$
\tilde{\mathcal{D}}_{00}\left(\mathbf{r}, \mathbf{r}^{\prime}, \omega\right)=\int d^{3} r_{1} d^{3} r_{2} \frac{1}{\left|\mathbf{r}-\mathbf{r}_{1}\right|} \Pi^{00}\left(\mathbf{r}_{1}, \mathbf{r}_{2}, \omega\right) \frac{1}{\left|\mathbf{r}_{2}-\mathbf{r}^{\prime}\right|}
$$

Performing the integrations leads to the result

$$
\tilde{\mathcal{D}}_{00}\left(\mathbf{r}, \mathbf{r}^{\prime}, \omega\right)=\sum_{L, M} B(E L ; L \rightarrow 0) \frac{2 \omega_{L}}{\omega^{2}-\omega_{L}^{2}-i \eta} \tilde{F}_{L}(r) \tilde{F}_{L}\left(r^{\prime}\right) Y_{L M}(\Omega) Y_{L M}^{*}\left(\Omega^{\prime}\right)
$$

where the radial dependence is carried by the functions

$$
\tilde{F}_{L}(r)=\frac{4 \pi}{(2 L+1) R_{0}^{L}}\left(\Theta\left(R_{0}-r\right) \frac{r^{L}}{R_{0}^{L+1}}+\Theta\left(r-R_{0}\right) \frac{R_{0}^{L}}{r^{L+1}}\right) .
$$

This form of the propagator explicitly reveals how observables like nuclear excitation energies $\omega_{L}$ and corresponding reduced electric transition probabilities come into play. The assumption of a sharp nuclear surface made here is reflected in the specific form of the radial behavior of the functions $\tilde{F}_{L}(r)$. However, it turns out that in contrast to the situation in muonic atoms, where the details of the $r$ dependence have significant influence on the results for the energy shift, only qualitative features (strongly pronounced maximum near the nuclear surface and regularity) are of importance in the case of electronic atoms.

Given the result for the harmonic surface vibrator one can immediately conclude the form of $\tilde{\mathcal{D}}_{00}$ in the case of a free nuclear collective rotator. Following similar steps as before (with respect to the body-fixed nuclear coordinate system) only minor changes are required due to a finite ground-state deformation described by an angular-dependent equilibrium radius $R_{0}(\Omega)$. Let $R_{i}=\min \left[R_{0}(\Omega)\right]$ and $R_{e}=\max \left[R_{0}(\Omega)\right]$, respectively; one obtains a radial function

$$
\tilde{F}_{L}(r)=\frac{4 \pi}{(2 L+1) R_{0}^{L+1}} \times \begin{cases}\frac{r^{L}}{R_{0}^{L+1}} & \text { if } 0 \leq r \leq R_{i} \\ \frac{R_{0}^{L}}{r^{L+1}} & \text { if } r \geq R_{e}\end{cases}
$$

being piecewise defined in regions inside a sphere with radius $R_{i}$ equal to the length of the smaller nuclear semiaxis and outside of a sphere with radius $R_{e}$ equal to that of the larger semiaxis, respectively. Based on (29) the easiest way to proceed further (but nevertheless sufficient for our purposes) is to interpolate the region $R_{i} \leq r \leq R_{e}$ by continuing the functional dependence given in (29) up to an average equilibrium radius $R_{0}^{\prime}$. This may legitimize why we can keep the same functions $\tilde{F}_{L}$ as obtained for the harmonic vibrator. The nuclear radii $R_{0}$ and $R_{0}^{\prime}$ will both be determined via $R_{0}=r_{0} A^{1 / 3}$ (with $r_{0}=1.2 \mathrm{fm}$ ). Again we note that a more careful analysis is adequate in 
the case of muonic atoms (compare with the interpolation proposed in Ref. 5).

A second modification is that the set of intermediate states $|\nu\rangle$ to be inserted in (23) is now given by eigenstates of the free rotation-vibration Hamiltonian $|\nu\rangle=\left|I M K n_{0} n_{2}\right\rangle \cdot{ }^{19}$ Evaluating the matrix elements $\left\langle 0\left|\hat{Q}_{L M}\right| I M K 00\right\rangle$ leads to

$$
\begin{array}{r}
\tilde{\mathcal{D}}_{00}\left(\mathbf{r}, \mathbf{r}^{\prime}, \omega\right)=\sum_{M, K} \frac{2 \omega_{2 K}}{\omega^{2}-\omega_{2 K}^{2}+i \eta} B(E 2 ; 2 K \rightarrow 0) \\
\times \tilde{F}_{2}(r) \tilde{F}_{2}\left(r^{\prime}\right) Y_{2 M}(\Omega) Y_{2 M}^{*}\left(\Omega^{\prime}\right),
\end{array}
$$

for quadrupole deformed nuclei. $K=0$ takes into account virtual excitations into the $2^{+}$state of the $0^{+}$ ground-state band while $K=2$ describes the (suppressed) transition from $2_{\gamma}^{+} \rightarrow 0_{G S}^{+}$. In actual calculations we will use experimental values for the transition probabilities and energies of the states taken into account.

Let us now derive the correction $\tilde{\mathcal{D}}_{00}$ to the propagator in the case of virtual excitations of giant resonances. We exploit the hydrodynamical description according to Steinwedel and Jensen. To begin with, we briefly review some basics of the model for spherical nuclei. ${ }^{19}$ The normal mode decomposition of the operator for proton density fluctuations reads

$$
\hat{\rho}_{\text {fluc }}(\mathbf{r}, t)=\rho_{0} \sum_{n, L, M} \hat{q}_{L M}^{(n) \dagger}(t) \eta_{L M n}(\mathbf{r})
$$

where

$$
\eta_{L M n}(\mathbf{r}, t)=N_{L n} j_{L}\left(k_{L n} r\right) Y_{L M}(\Omega)
$$

are the regular eigenfunctions of the Helmholtz equation. The wave number $k_{L n}$ of the $n$th harmonic with multipolarity $L$ is determined by the requirement of vanishing proton current through the nuclear surface (equilibrium sphere with radius $R_{0}$ ) by imposing the Neumann condition

$$
\left.\left(\frac{d}{d r} j_{L}\left(k_{L n} r\right)\right)\right|_{r=R_{0}}=0
$$

We note the first zeros $z_{L n}=k_{L n} R_{0}$ which will be used in later calculations:

$$
z_{11}=2.08, \quad z_{21}=3.34, \quad z_{31}=4.51 .
$$

The normalization factor $N_{L_{n}}$ follows from the normalization condition:

$$
N_{L n}=\left\{\frac{1}{2} R_{0}^{3}\left[j_{L}^{2}\left(z_{L n}\right)-j_{L-1}\left(z_{L n}\right) j_{L+1}\left(z_{L n}\right)\right]\right\}^{-\frac{1}{2}}
$$

with $z_{L n}=k_{L n} R_{0}$. Performing now similar steps as indicated above we can write down the polarization function

$$
\begin{aligned}
\Pi^{00}\left(\mathbf{r}, \mathbf{r}^{\prime}, \omega\right)=\rho_{0}^{2} \sum_{n, L, M} \sum_{n^{\prime}, L^{\prime}, M^{\prime}} \sum_{n^{\prime \prime}, L^{\prime \prime}, M^{\prime \prime}} & \frac{2 \omega_{L^{\prime \prime} n^{\prime \prime}}}{\omega^{2}-\omega_{L^{\prime \prime} n^{\prime \prime}}^{2}+i \eta} \\
& \times \eta_{L M n}^{*}(\mathbf{r}) \eta_{L^{\prime} M^{\prime} n^{\prime}}\left(\mathbf{r}^{\prime}\right)\left\langle 0\left|\hat{q}_{L M}^{(n)}\right| n^{\prime \prime} L^{\prime \prime} M^{\prime \prime}\right\rangle\left\langle n^{\prime \prime} L^{\prime \prime} M^{\prime \prime}\left|\hat{q}_{L^{\prime} M^{\prime}}^{\left(n^{\prime}\right) \dagger}\right| 0\right\rangle
\end{aligned}
$$

which is the analogous expression to (23) now evaluated by inserting a complete set of eigenstates

$$
|\nu\rangle=|n L M\rangle=\hat{\beta}_{L M}^{(n) \dagger}|0\rangle
$$

of the collective Hamiltonian

$$
\hat{H}_{\mathrm{GR}}=\sum_{n, L, M} \omega_{L n}\left(\hat{\beta}_{L M}^{(n) \dagger} \hat{\beta}_{L M}^{(n)}+\frac{1}{2}\right)
$$

for giant resonances. Introducing electric multipole operators via

$$
\hat{Q}_{L M}(t)=\int d^{3} r r^{L} Y_{L M}^{*}(\Omega) \hat{\rho}_{\mathrm{fluc}}(\mathbf{r}, t)=\sum_{n} \hat{Q}_{L M}^{(n)}
$$

where

$$
\hat{Q}_{L M}^{(n)}=\rho_{0} N_{L n} G_{l n} \hat{q}_{L M}^{(n)}, \quad G_{L n}=R_{0}^{L+3} \frac{j_{L+1}\left(z_{L n}\right)}{z_{L n}}
$$

denotes the contribution of a single harmonic (labeled by the index $n$ ) we can write

$$
\Pi^{00}\left(\mathbf{r}, \mathbf{r}^{\prime}, \omega\right)=\sum_{n, L, M} \frac{2 \omega_{L n}}{\omega^{2}-\omega_{L n}^{2}+i \eta}\left|\left\langle 0\left|\hat{Q}_{L M}^{(n)}\right| n L M\right\rangle\right|^{2} G_{L n}^{-2} j_{L}\left(k_{L n} r\right) j_{L}\left(k_{L n} r^{\prime}\right) Y_{L M}(\Omega) Y_{L M}^{*}\left(\Omega^{\prime}\right)
$$

Computing again the effective propagator according to (26) leads to the result

$$
\tilde{\mathcal{D}}_{00}\left(\mathbf{r}, \mathbf{r}^{\prime}, \omega\right)=\sum_{n, L, M} \frac{2 \omega_{L n}}{\omega^{2}-\omega_{L n}^{2}+i \eta} \tilde{\mathcal{F}}_{L}^{(n)}(r) \tilde{\mathcal{F}}_{L}^{(n)}\left(r^{\prime}\right) Y_{L M}(\Omega) Y_{L M}^{*}\left(\Omega^{\prime}\right),
$$


with radial functions

$$
\begin{aligned}
\tilde{\mathcal{F}}_{L}^{(n)}(r)=\frac{4 \pi}{R_{0}^{L}(2 L+1)}\{ & \Theta\left(R_{0}-r\right) \frac{r^{L}}{R_{0}^{L+1}}+\Theta\left(r-R_{0}\right) \frac{R^{L}}{r^{L+1}} \\
& \left.+\Theta\left(R_{0}-r\right) \frac{2 L+1}{R_{0} z_{L n}}\left[\frac{j_{L}\left(z_{L n} r / R_{0}\right)}{j_{L+1}\left(z_{L n}\right)}-\left(\frac{r}{R_{0}}\right)^{L} \frac{j_{L}\left(z_{L n}\right)}{j_{L+1}\left(z_{L n}\right)}\right]\right\} .
\end{aligned}
$$

Notice that the $\tilde{\mathcal{F}}_{L}^{(n)}$ contain a part which does not depend on $n$. It is identical to the radial functions $\tilde{F}_{L}$ obtained for the surface vibrator. The second part in $\tilde{\mathcal{F}}_{L}^{(n)}$ causes slight changes. It shifts the maximum towards the interior of the nucleus (see figures in Sec. III).

The question arises whether the convergence of the infinite summation over all harmonics $n$ for fixed multipolarity $L$ is a priori guaranteed. A minor consideration reveals that this is the case. To give a simple argument one may look at the behavior of each term contributing to $\tilde{\mathcal{D}}_{00}$ for large values of $z_{L n}$ [ $L$ fixed; suppose $z_{L n} \sim$ const $(L)+n$ for $n \gg 1$ ]. For large $n$ we expect roughly the following asymptotic behavior of the relevant factors:

$$
\begin{aligned}
& \left|\left\langle 0\left|\hat{Q}_{L M}^{(n)}\right| L M\right\rangle\right|^{2} \sim \frac{1}{z_{L n}^{2}}, \\
& \tilde{\mathcal{F}}_{L}^{(n)}(r) \sim \tilde{F}_{L}(r)+O\left(1 / z_{L n}\right) .
\end{aligned}
$$

Together with an overall factor $1 / \omega_{L n}$ from the energy denominator we conclude that these terms will decrease sufficiently rapidly for $n \gg 1$. For reasons of simplicity let us assume that resonance states with fixed multipolarity $L$ but different $n$ (if such states really exist) may be properly described by one state $|L M 1\rangle$ with an average energy $\bar{\omega}_{L} \equiv \omega_{L 1}$. This also implies the introduction of average transition probabilities according to

$$
\begin{aligned}
\sum_{n} \mid\left\langle 0\left|\hat{Q}_{L M}^{(n)}\right|\right. & L M n\rangle\left.\right|^{2} \delta\left(\omega^{\prime}-\omega_{L n}\right) \\
& \rightarrow\left|\left\langle 0\left|\hat{Q}_{L M}\right| L M 1\right\rangle\right|^{2} \delta\left(\omega^{\prime}-\bar{\omega}_{L}\right)
\end{aligned}
$$

Under these assumptions we can further simplify the expression for the propagator, which now again takes the form

$$
\begin{aligned}
\tilde{\mathcal{D}}_{00}\left(\mathbf{r}, \mathbf{r}^{\prime}, \omega\right)=\sum_{L, M} & \frac{2 \bar{\omega}_{L}}{\omega^{2}-\bar{\omega}_{L}^{2}+i \eta} B(E L ; L \rightarrow 0) \\
& \times \tilde{\mathcal{F}}_{L}^{(1)}(r) \tilde{\mathcal{F}}_{L}^{(1)}\left(r^{\prime}\right) Y_{L M}(\Omega) Y_{L M}^{*}(\Omega),
\end{aligned}
$$

where $B(E L ; L \rightarrow 0)$ stands for an average reduced transition probability. In practical calculations we will use empirical data.

For the sake of completeness let us briefly mention that by means of similar arguments as used before one can derive the correction to the propagator for monopole excitations. Based on the Goldhaber-Tassie form of the transition density

$$
\hat{\rho}_{\text {fluc }}(\mathbf{r}, t)=-\left(r \frac{d \rho_{0}}{d r}+3 \rho_{0}(r)\right) \hat{\alpha}_{00}^{\dagger}(t) Y_{00}(\Omega),
$$

and assuming $\rho_{0}(r)=\rho_{0} \Theta\left(R_{0}-r\right)$ to be the nuclear charge density in its ground state the resulting modification of the propagator $\tilde{\mathcal{D}}_{00}$ is given by

$$
\begin{aligned}
& \tilde{\mathcal{D}}_{00}\left(\mathbf{r}, \mathbf{r}^{\prime}, \omega\right)=B(E 0 ; 0 \rightarrow 0) \frac{2 \omega_{0}}{\omega^{2}-\omega_{0}^{2}+i \eta} \tilde{F}_{0}(r) \tilde{F}_{0}\left(r^{\prime}\right), \\
& \tilde{F}_{0}(r)=\frac{1}{R_{0}^{2}} \Theta\left(R_{0}-r\right)\left[1-\left(\frac{r}{R_{0}}\right)^{2}\right] .
\end{aligned}
$$

We also employed the (phenomenological) energy weighted sum rule

$$
\sum_{f} \omega_{f} B(E 0 ; 0 \rightarrow f)=\alpha \frac{15 Z^{2} R_{0}^{2}}{m_{N} A},
$$

and it was assumed that it is completely fulfilled by one state with excitation energy $\omega_{0}$ and reduced transition probability $B(E 0)$.

\section{Evaluation of the effective self-energy}

Having derived explicit expressions for the longitudinal component of the effective propagator we are now in the position to evaluate the first-order self-energy contribution to the energy of atomic bound states. According to (15) we have to insert the exact electron propagator $S_{F}\left(\mathbf{x}, \mathbf{x}^{\prime}, E\right)$ for electrons in the external field of a homogeneously charged sphere. Since an analytical expression is not available we formally make use of its eigenfunction expansion into Furry states:

$$
S_{F}\left(\mathbf{x}, \mathbf{x}^{\prime}, E\right)=\sum_{k} \frac{\psi_{k}(\mathbf{x}) \bar{\psi}_{k}\left(\mathbf{x}^{\prime}\right)}{E-E_{k}-i \bar{\eta}}
$$

where we will use the convenient spherical representation of the eigenfunctions $\psi_{k}:{ }^{21}$

$$
\begin{aligned}
& \psi_{k}(\mathbf{r}) \equiv \psi_{\nu_{1} \kappa_{1} \mu_{1}}(\mathbf{r})=\frac{1}{r}\left(\begin{array}{c}
g_{\nu_{1} \kappa_{1}}(r) \chi_{\kappa_{1} \mu_{1}}(\Omega) \\
i f_{\nu_{1} \kappa_{1}}(r) \chi_{\kappa_{1}-\mu_{1}}(\Omega)
\end{array}\right) \\
& \kappa_{1}=\left\{\begin{array}{l}
\ell_{1} \text { if } j_{1}=\ell_{1}-\frac{1}{2} \\
-\left(\ell_{1}+1\right) \text { if } j_{1}=\ell_{1}+\frac{1}{2}
\end{array}\right.
\end{aligned}
$$

$\nu_{1}$ stands either for the principal quantum number of a bound state or the energy of a continuum state. Introducing the following quantities (let $F_{L}$ denote any radial function occurring in $\tilde{\mathcal{D}}_{00}$ ): 


$$
\begin{aligned}
\mathcal{R}_{\nu \kappa \mid \nu_{1} \kappa_{1}}^{L} & =\int_{0}^{\infty} d r F_{L}(r)\left[g_{\nu \kappa}(r) g_{\nu_{1} \kappa_{1}}(r)+f_{\nu \kappa}(r) f_{\nu_{1} \kappa_{1}}(r)\right] \\
& =\left\langle\nu \kappa\left|F_{L}\right| \nu_{1} \kappa_{1}\right\rangle \\
\mathcal{E}_{\nu \kappa \mid \nu_{1} \kappa_{1}}^{L} & =\int_{-\infty}^{\infty} d E\left(E-E_{\nu_{1} \kappa_{1}}+i \bar{\eta}\right)^{-1} \frac{2 E_{L}}{\left(E-E_{\nu \kappa}\right)^{2}-E_{L}^{2}+i \tilde{\eta}} \\
\mathcal{W}_{\kappa \mu \mid \kappa_{1} \mu_{1}}^{L} & =\int d \Omega Y_{L M}(\Omega) \chi_{\kappa \mu}^{+}(\Omega) \chi_{\kappa_{1} \mu_{1}}(\Omega)=\left\langle\kappa \mu\left|Y_{L M}\right| \kappa_{1} \mu_{1}\right\rangle
\end{aligned}
$$

the self-energy can be cast into the form

$$
\Delta E_{\nu \kappa \mu}=i \alpha \sum_{L} B(E L ; L \rightarrow 0) \sum_{\kappa_{1}} \sum_{M, \mu_{1}}\left(\mathcal{W}_{\kappa \mu \mid \kappa_{1} \mu_{1}}^{L M}\right)^{2} \int_{\nu_{1}} \mathcal{E}_{\nu \kappa \mid \nu_{1} \kappa_{1}}^{L}\left(\mathcal{R}_{\nu \kappa \mid \nu_{1} \kappa_{1}}^{L}\right)^{2}
$$

The above equations already indicate the order in which summations and integrations will be carried out. Questions about the convergence of this formal expression will be addressed later.

The angular matrix elements are straightforward to calculate leading to the result

$$
\sum_{M, \mu_{1}}\left(\mathcal{W}_{\kappa \mu \mid \kappa_{1} \mu_{1}}^{L M}\right)^{2}=\frac{(-1)^{2 \mu-1}}{2 \pi}\left|\kappa_{1}\right|\left(j_{1} j L \mid \frac{1}{2}-\frac{1}{2} 0\right)^{2}, \quad j_{1}=\left|\kappa_{1}\right|-\frac{1}{2},
$$

where we have suppressed the triangle relations $\delta\left(\ell \frac{1}{2} j\right) \delta\left(\ell_{1} \frac{1}{2} j_{1}\right) \delta\left(j_{1} j L\right)$ as well as a parity factor:

$$
P\left(\ell, \ell_{1}, L\right)=\frac{1}{4}\left[1+(-1)^{\ell+\ell_{1}+L}\right]^{2}= \begin{cases}1 & \text { if } \ell+\ell_{1}+L=\text { even } \\ 0 & \text { otherwise }\end{cases}
$$

For evaluating $\mathcal{E}_{\nu \kappa \mid \nu_{1} \kappa_{1}}^{L}$ the integration over energy can be performed by means of complex contour integration (e.g., closing the contour in the lower half plane):

$$
\begin{aligned}
\mathcal{E}_{\nu \kappa \mid \nu_{1} \kappa_{1}}^{L} & =\int_{-\infty}^{\infty} d E\left(E-E_{\nu_{1} \kappa_{1}}+i \bar{\eta}\right)^{-1}\left[\left(E-E_{\nu \kappa}-E_{L}+i \tilde{\eta}^{\prime}\right)^{-1}-\left(E-E_{\nu \kappa}+E_{L}-i \tilde{\eta}^{\prime}\right)^{-1}\right] \\
& =2 \pi i\left(\frac{\Theta\left(E_{\nu_{1} \kappa_{1}}-E_{F}\right)}{E_{\nu_{1} \kappa_{1}}-E_{\nu \kappa}+E_{L}}+\frac{\Theta\left(E_{F}-E_{\nu_{1} \kappa_{1}}\right)}{E_{\nu_{1} \kappa_{1}}-E_{\nu \kappa}-E_{L}}\right)
\end{aligned}
$$

Inserting these results into (49) we end up with the general expression for the effective self-energy:

$$
\begin{aligned}
& \Delta E_{\nu \kappa \mu}=-\alpha \sum_{L, \kappa_{1}} B(E L ; L \rightarrow 0)\left|\kappa_{1}\right|\left(j_{1} j L \mid \frac{1}{2}-\frac{1}{2} 0\right)^{2} \mathcal{M}_{\nu \kappa \mid \kappa_{1}}^{L}, \\
& \mathcal{M}_{\nu \kappa \mid \kappa_{1}}^{L}=\int_{1}^{\infty} d E^{\prime} \frac{\left|\left\langle\nu \kappa\left|F_{L}\right| E^{\prime} \kappa_{1}\right\rangle\right|^{2}}{E^{\prime}-E_{\nu \kappa}+E_{L}}+\sum_{\nu_{1}} \frac{\left|\left\langle\nu \kappa\left|F_{L}\right| \nu_{1} \kappa_{1}\right\rangle\right|^{2}}{E_{\nu_{1} \kappa_{1}}-E_{\nu \kappa}+E_{L}-i \eta}+\int_{-\infty}^{-1} d E^{\prime} \frac{\left|\left\langle\nu \kappa\left|F_{L}\right| E^{\prime} \kappa_{1}\right\rangle\right|^{2}}{E^{\prime}-E_{\nu \kappa}-E_{L}} .
\end{aligned}
$$

The overall minus sign of the energy shift indicates that this part of the nuclear polarization increases the binding energy of electronic states. It parametrically depends on the excitation energies $E_{L}$ and the corresponding $B(E L)$ values. The first two terms in $\mathcal{M}_{\nu \kappa \mid \kappa_{1}}^{L}$ are equivalent to the usual expressions derived in second-order perturbation theory. They mean that the considered electron in the bound state $|\nu \kappa \mu\rangle$ can be excited into a higher unoccupied intermediate state $\left|\nu_{1} \kappa_{1}\right\rangle$ by a virtual (effective) photon. The third term has to be understood in the charge-conjugated picture where a hole can be demoted into the negative-energy continuum by emission of a photon. This vacuum contribution has not been taken into account in previous calculations. The discrete summation over the bound states needs some special attention.
For an excited electron state $|\nu \kappa \mu\rangle$ the energy difference $\left(E_{\nu_{1} \kappa_{1}}-E_{\nu \kappa}+E_{L}\right)$ can become arbitrarily small reflecting the possibility of spontaneous decay into a lower-lying unoccupied bound state. Close to such resonances the perturbative treatment of the energy shift is in general no longer adequate. However, using perturbation theory we can estimate the spontaneous transition probability as the imaginary part of the level energy of a bound state. It gives rise to a finite width of the excited state due to the interaction with the radiation field. ${ }^{22}$ In a consistent calculation no divergencies appear. It should also be emphazised that such a resonance may only occur in connection with low-lying nuclear rotational states. On the other hand, one can calculate matrix elements $\left\langle\nu \kappa\left|F_{L}\right| \nu_{1} \kappa_{1}\right\rangle$ and compare them with those involv- 
ing continuum states. The latter turn out to be orders of magnitude larger. One reason lies in the radial functions $F_{L}$ which are predominantly peaked near the nuclear surface where the overlap between different bound states is small by itself. For that reason we will later neglect the contribution to the energy shift arising from the sum over bound states. Further aspects concerning the convergence of the integration over the continuum will be discussed in the following section.

\section{NUCLEAR-POLARIZATION CONTRIBUTION TO THE BINDING ENERGY OF $K$-SHELL ELECTRONS}

In the preceding section we have explained how to treat nuclear-polarization contributions as effective radiative corrections. Having derived the general formula for the effective self-energy in first-order perturbation theory we are now ready for specific applications. To demonstrate the importance of the effect and the practicability of our approach we will calculate the contribution to the $1 s$ Lamb shift in hydrogenlike lead and uranium. It is the latter which is the focus of various planned experiments because it has the highest accessible nuclear charge. We will use available experimental data for the various nuclear excitations taken into account. The model calculation for a superheavy system will representatively illustrate the general features and the increase of nuclearpolarization effects for large $Z$.

\section{A. Some numerical details}

The practicality of solving Eq. (53) for the energy shift may be best shown by a brief discussion of the main aspects encountered with the numerical evaluation. The general procedure will be the following: The contribution of each single nuclear excitation (with multipolarity $L)$ to the energy shift (53), denoted by $\Delta E_{\nu \kappa \mu}^{L}$, has to be calculated separately. For a given type of nuclear excitation (vibrations, rotations, giant resonances) with multipolarity $L$ we first have to compute the radial matrix elements $\left\langle\nu \kappa\left|F_{L}\right| E \kappa_{1}\right\rangle$ between the considered electron bound state $|\nu \kappa\rangle$ and the intermediate continuum state $\left|E \kappa_{1}\right\rangle$ with the radial function $F_{L}$ of the corresponding propagator. The allowed values for $\kappa_{1}$ follow from the selection rules implied by $(50)$. The dependence of $\left|\left\langle\nu \kappa\left|F_{L}\right| E \kappa_{1}\right\rangle\right|^{2}$ on the energy $E$ of the continuum state will tell us how far we have to integrate to ensure convergence. The resulting quantities $\tilde{\mathcal{M}}_{\nu \kappa \mid \kappa_{1}}^{L}$ (the tilde indicates that we neglect the contribution from the summation over the bound states) depending on the excitation energy $E_{L}$ have then to be multiplied with the corresponding $B(E L)$ values. In a last step one has to perform the summation over all intermediate angular momentum quantum numbers $\kappa_{1}$ to obtain the contribution $\Delta E_{\nu \kappa \mu}^{L}$ for a given nuclear multipole $L$.

To begin with, let us compare the radial functions $F_{L}$ in the case of vibrations (rotations) (see Fig. 1) and gi-

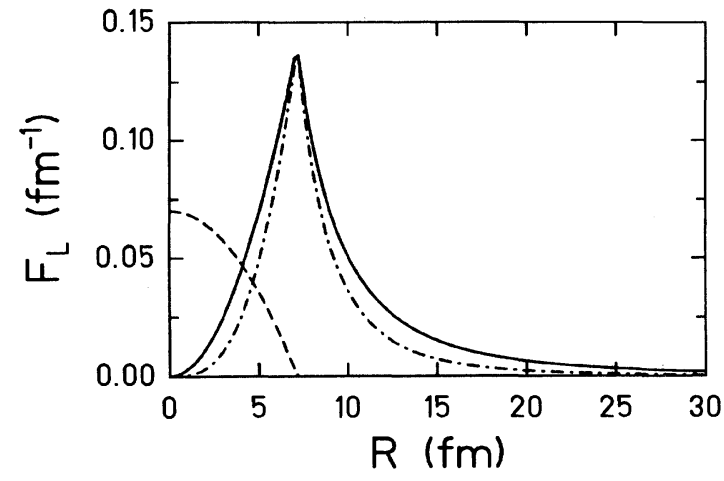

FIG. 1. Radial functions for collective vibrations and rotations ( $L=0$, dashed line; $L=2$, solid line; $L=3$, dashdotted line). $\tilde{F}_{L}(2 L+1) R_{0}^{L} / 4 \pi$ is plotted.

ant resonances (see Fig. 2). As a common feature of both types of functions they exhibit a pronounced maximum near the nuclear surface. Strictly speaking, while the maximum is located at $r=R_{0}$ in the case of surface vibrations it is slightly shifted towards the interior of the nucleus in the case of giant resonaces. They both decrease with the same power law $\sim r^{-(L+1)}$ outside the nucleus. It is exactly this decrease of $F_{L}$ which additionally supports convergence of the radial matrix elements. Even if one would like to calculate the energy shift of higher bound states a sufficiently large integration interval is found to be $0 \leq r \leq r_{\max } \sim 10^{4} \mathrm{fm}$. We perform the integration using a Gauss-Legendre routine with adjustable number of grid points. For increasing continuum energy the corresponding radial functions $g_{E \kappa_{1}}$ and $f_{E \kappa_{1}}$ oscillate strongly. To ensure a sufficient accuracy even for high continuum energies the radial integration is designed according to the following scheme: We divide the total interval $I=\left[0, r_{\max }\right]$ into three principal intervals $I_{1}=\left[0, R_{0}\right], I_{2}=\left[R_{0}, r_{0}\right]\left(\right.$ with $\left.r_{0} \sim 100 \mathrm{fm}\right)$, and $I_{3}=\left[r_{0}, r_{\max }\right]$. Regarding the fact that the functions $F_{L}$

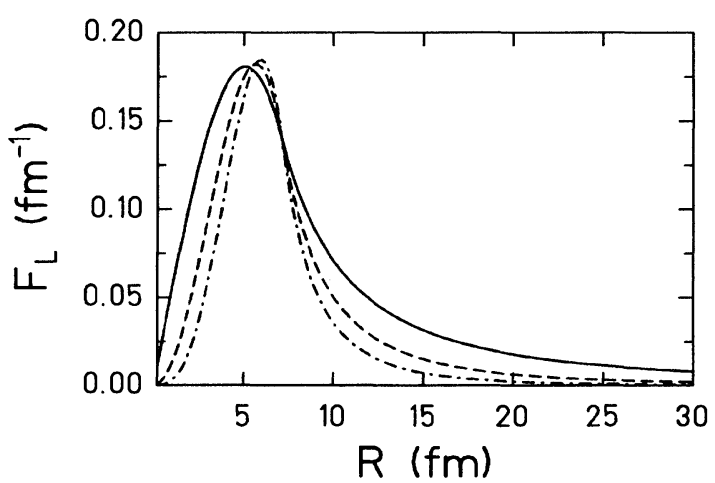

FIG. 2. Radial functions for giant reson ances $(L=1$, solid line; $L=2$, dashed line; $L=3$, dash-dotted line). $\tilde{\mathcal{F}}_{L}(2 L+$ 1) $R_{0}^{L} / 4 \pi$ is plotted. 
have their pronounced maximum near the nuclear surface which we like to represent well, the first two intervals cover the interior and the near outside region of the nucleus. The exterior region $I_{3}$ is divided into a number of $N$ subintervals of equal size where $N$ can be large depending on the energy of the continuum state. Performing the integration over each subinterval using again a Gauss-Legendre routine one may now determine $N$ by the requirement of a minimum number of grid points per half oscillation of the continuum state. Proceeding in that way it is possible to achieve an accuracy for the matrix element $\left\langle\nu \kappa\left|F_{L}\right| E \kappa_{1}\right\rangle$ of about $10^{-8}$ up to continuum energies $E \sim 750 \mathrm{MeV}$.

As the next step towards the integration over continuum energies we have to investigate the energy dependence of the matrix elements squared, $\left|\left\langle\nu \kappa\left|F_{L}\right| E \kappa_{1}\right\rangle\right|^{2}$. An extensive analysis of such matrix elements reveals that they all display similar characteristic features. As a function of $E$ they show an oscillatory behavior which becomes strongly suppressed with increasing $|E|^{2}$. Figure 3 displays a typical matrix element in connection with quadrupole vibrations in ${ }_{82}^{208} \mathrm{~Pb}$. It has a dominant maximum for positive continuum energies at $E \sim 50 \mathrm{MeV}$, i.e., the main contribution arises due to virtual excitations into the unoccupied electron continuum. Additionally there is a less pronounced maximum (and also smaller in magnitude) in the negative-energy continuum. The basic character of the energy dependence can be understood qualitatively from the fact that, roughly speaking, the matrix elements $\left\langle\nu \kappa\left|F_{L}\right| E \kappa_{1}\right\rangle$ are Fourier-Bessel transforms of the radial functions $F_{L}$. The oscillations are due to the strongly localized peaklike structure. Detailed studies support the expectation that the position of the maximum remains almost at the same position even for higher multipoles $L$ and for larger values of $Z$. Its magnitude, of course, increases with $Z$.

Compared to these extrema the others appearing for large $|E|$ are strongly damped and thus of minor importance. In order to convince ourselves we calculated

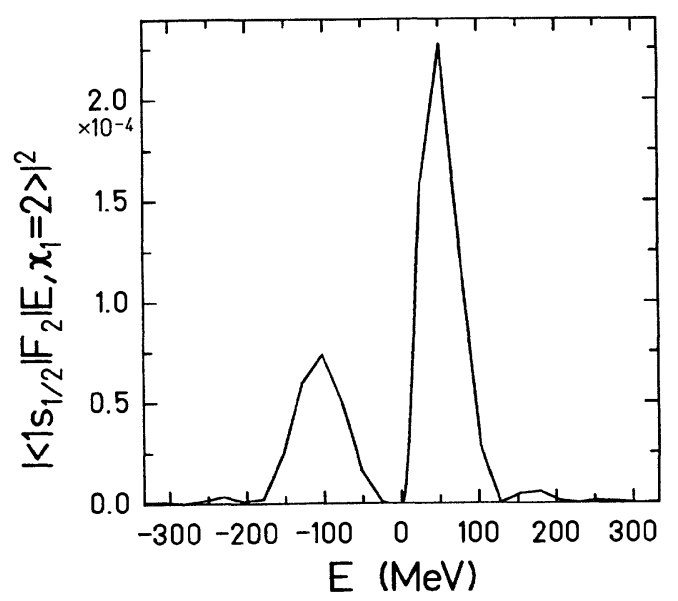

FIG. 3. The matrix element $\left|\left\langle 1 s_{1 / 2}\left|F_{L}\right| E \kappa_{1}=2\right\rangle\right|^{2}$ for ${ }_{82}^{208} \mathrm{~Pb}$. matrix elements up to continuum energies $|E| \sim 750$ MeV. A semilogarithmic plot (see Fig. 4) of a typical matrix element over the considered energy range reveals a sufficiently rapid decrease in magnitude. Usually we found convergence of the energy integration (performed by means of a Gauss-Legendre routine) for energies $|E|$ less than $350 \mathrm{MeV}$. Note that in addition the energy denominators in $\tilde{\mathcal{M}}_{\nu \kappa \mid \kappa_{1}}^{L}$ improve the convergence. Fixing the upper integration limits at $|E| \simeq 350 \mathrm{MeV}$ ensured a relative accuracy up to $10^{-4}$. It should be mentioned that corresponding matrix elements calculated with the function $\mathcal{F}_{L}$ for giant resonances show the same qualitative features because of the similarities between the functions $F_{L}$ and $\mathcal{F}_{L}$.

Based on these feasibility studies we conclude: The direct evaluation of the self-energy (53) using an eigenfunction decomposition of the electron propagator $S_{F}$ is practical. Some effort has to be spent in performing the radial integration with sufficient accuracy for large continuum energies. The specific form of the structure functions $F_{L}$ supports convergence of both the radial and the energy integration. In addition, they guarantee that contributions from higher multipoles become increasingly less important. As a consequence also the absolute convergence of the summation over angular momenta is ensured. In view of the properties implied by the effective propagator $\tilde{\mathcal{D}}_{00}$ the evaluation of the effective self energy in the manner proposed here is quite different from the situation encountered for the usual QED self-energy, where the energy integration is logarithmically divergent.

B. Nuclear-polarization contribution to the Lamb shift in lead and uranium

We now turn the discussion to the calculation of the effective self-energy shift of the $1 s$ bound state in hydrogenlike ${ }_{82}^{208} \mathrm{~Pb}$ and ${ }_{92}^{238} \mathrm{U}$. Both nuclei have well-established collective excitation modes. While the first is a candidate for a collective nuclear vibrator the latter allows one to study the effect of low-lying rotational states. To check our formalism let us first look at pure model calculations

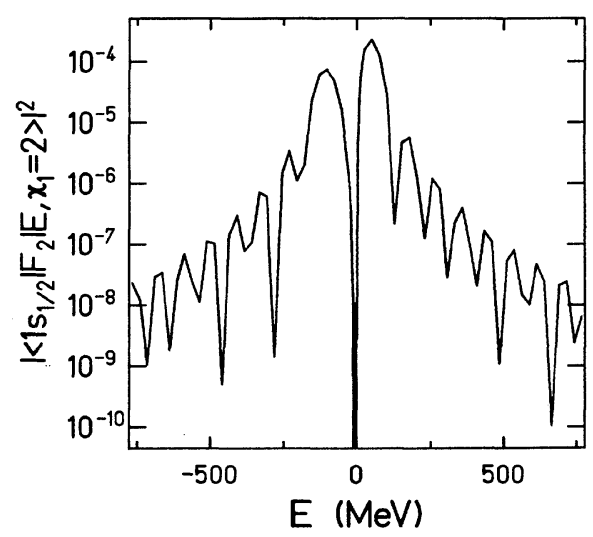

FIG. 4. Convergence of $\left|\left\langle 1 s_{1 / 2}\left|F_{2}\right| E \kappa_{1}\right\rangle\right|^{2}$. 
where we also use the analytical (model dependent) expressions for the $B(E L)$ values. We simply consider the contributions $\Delta E_{1 s}^{L}$ for vibrational modes as a representative example. This gives a first insight about the upper bound for the order of magnitude as well as the systematic dependence on the multipolarity $L$. The results for virtual monopole and quadrupole excitations for $Z=82$ and 92 are shown in Figs. 5(a) and 5(b), respectively. As expected, the contributions $\Delta E_{1 s}^{L}$ grow with increasing nuclear charge $Z$ but decrease with increasing multipolarity. The systematic increase of the effect for small excitation energies indicates the importance of including low-lying collective excitations. We therefore expect the energy shift to be larger in uranium than in lead. Model calculations performed in the case of giant resonances show similar dependences.

Let us now briefly comment on the more realistic results obtained for the nuclear-polarization effect on the $1 s$-Lamb shift based on experimantal values for the transition energies and the reduced transition probabilities. In the case of ${ }_{82}^{208} \mathrm{~Pb}$ we calculated the contributions caused by virtual excitation of the following modes: (a) the collective quadrupole excitation $\left(2^{+}\right.$state $)$at $E_{2}^{\text {vib }}=4.086 \mathrm{MeV}$ and the low-lying octupole excitation $\left(3^{-}\right.$state $)$at $E_{3}^{\text {vib }}=2.615 \mathrm{MeV}$, both with experimental $B(E L)$ values; $^{23}$ (b) the giant quadrupole resonance at $E_{2}^{\mathrm{GR}} \simeq 12 \mathrm{MeV}$ again with empirical data. ${ }^{24}$ For completeness we also calculated the contribution due to the monopole vibrational state at $E_{0}=13.5 \mathrm{MeV}$ and the dominant giant dipole resonance at $E_{1}^{\mathrm{GR}}=13.7 \mathrm{MeV}$ where the $B(E L)$ values have been taken from Ref. 25 . The results are displayed in Table I. They are in convincing agreement with values obtained earlier. ${ }^{8}$

We now turn to ${ }_{92}^{238} \mathrm{U}$ where we can study the effect of low-lying rotational states. The following $E 2$ transitions have been taken into account: (a) the transition $2_{\mathrm{GS}}^{+} \rightarrow 0_{\mathrm{GS}}^{+}$within the ground-state band $(K=0)$ (b) the transition between the $2_{\gamma}^{+}$state in the $\gamma$ band $(K=2)$ and the $0_{\mathrm{GS}}^{+}$state. This calculation is again based on experimental values for the reduced transition probabilities. ${ }^{26}$ The results are given in Table II. It contains additional results of a model calculation estimating the contributions of the giant dipole and the giant quadrupole resonance, respectively. The energy shift due to virtual excitation of the $2^{+}$rotational state of the ground-state band obviously dominates. It is about two
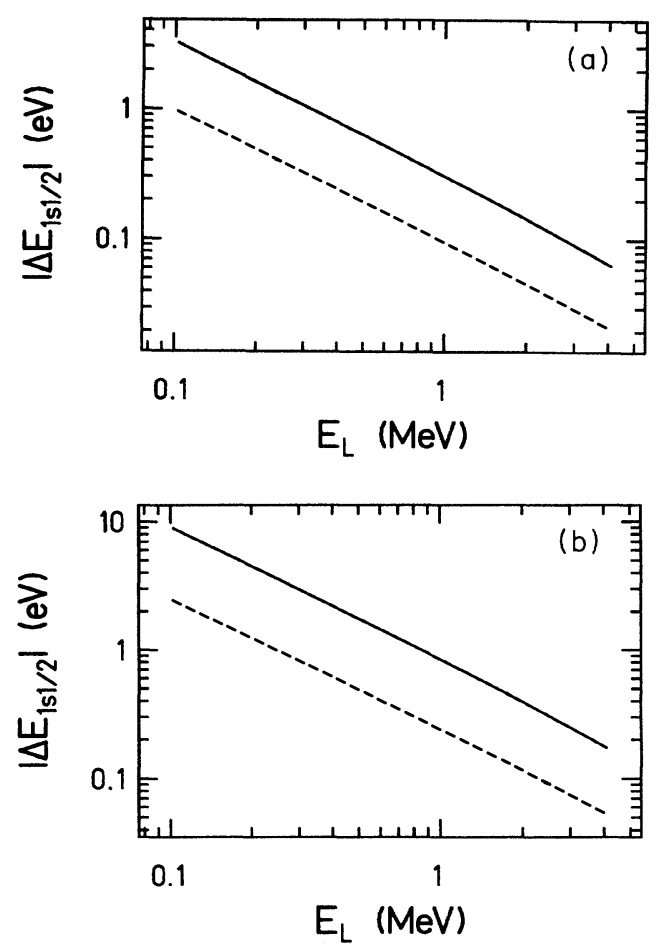

FIG. 5. (a) Contributions $\Delta E_{1 s_{1 / 2}}\left(Z=82, E_{L}\right)$ for $L=0$ (solid line) and $L=2$ (dashed line); (b) the same as in (a) for $Z=92$.

orders of magnitude larger than the one caused by the $E 2$ transition in ${ }_{82}^{208} \mathrm{~Pb}$, a fact which is not surprising in view of the large differences in the corresponding $B(E L)$ values. One should again incorporate the contributions arising from giant resonances. Let us mention that for the type of collective excitations considered here the results do not significantly depend on the explicit form of the functions $F_{L}$.

If we compare the nuclear-polarization contribution with the QED Lamb shift ${ }^{3}$ we find

$$
\frac{\left|\Delta E_{1 s_{1 / 2}}^{\mathrm{NP}}\right|}{\left|\Delta E_{1 s_{1 / 2}}^{\mathrm{QED}}\right|}=\left\{\begin{array}{l}
4 \times 10^{-4} \text { for }{ }_{82}^{208} \mathrm{~Pb} \\
2 \times 10^{-3} \text { for }{ }_{92}^{238} \mathrm{U}
\end{array}\right.
$$

At that point let us only comment on the influence of

TABLE I. Energy shift of the $1 s_{1 / 2}, 2 s_{1 / 2}$, and $2 p_{1 / 2}$ state due to various collective excitations in ${ }_{82}^{208} \mathrm{~Pb} . \Delta E^{L}$ is given in units of $\mathrm{meV}$.

\begin{tabular}{ccccc}
\hline \hline$E_{L}(\mathrm{MeV})$ & $B(E L ; L \rightarrow 0)\left(\right.$ units of $\left.e^{2} \mathrm{~b}^{L}\right)$ & $\left|\Delta E_{1 s}^{L}\right|$ & $\left|\Delta E_{2 s}^{L}\right|$ & $\left|\Delta E_{2 p_{1 / 2}}^{L}\right|$ \\
\hline$E_{0}=13.5$ & 0.199 & 23.7 & 4.1 & 0.3 \\
$E_{1}^{\mathrm{GR}}=13.7$ & 0.072 & 47.0 & 8.1 & 0.7 \\
$E_{2}^{\mathrm{GR}}=12.0$ & 0.106 & 16.9 & 2.9 & 0.2 \\
$E_{2}^{\text {vib }}=4.086$ & 0.060 & 8.0 & 1.4 & 0.1 \\
$E_{3}^{\text {vib }}=2.615$ & 0.096 & 5.7 & 0.9 & 0.1 \\
\hline \hline
\end{tabular}


TABLE II. Energy shift of the $1 s_{1 / 2}, 2 s_{1 / 2}$, and $2 p_{1 / 2}$ state due to low-lying rotational states and due to giant resonances (GR) in ${ }_{92}^{23}$ U. $\Delta E^{L}$ is given in units of meV.

\begin{tabular}{cccccc}
\hline \hline transition & $E(\mathrm{MeV})$ & $B(E L ; L \rightarrow 0)\left(\right.$ units of $\left.e^{2} \mathrm{~b}^{L}\right)$ & $\left|\Delta E_{1 s}^{L}\right|$ & $\left|\Delta E_{2 s}^{L}\right|$ & $\left|\Delta E_{2 p_{1 / 2}}^{L}\right|$ \\
\hline $2_{\mathrm{GS}}^{+} \rightarrow 0_{\mathrm{GS}}^{+}$ & 0.0411 & 2.38 & 808.0 & 151.8 & 17.5 \\
$2_{\gamma}^{+} \rightarrow 0_{\mathrm{GS}}^{+}$ & 1.0583 & 0.025 & 7.7 & 1.4 & 0.2 \\
$\mathrm{GR}(L=1)$ & 13.0 & 0.135 & 195.1 & 36.6 & 4.2 \\
$\mathrm{GR}(L=2)$ & 10.0 & 0.195 & 68.4 & 12.9 & 1.5 \\
\hline \hline
\end{tabular}

the rotational state on the $2 s_{1 / 2}-2 p_{1 / 2}$ Lamb shift in uranium. Nuclear-polarization effects tend to reduce the $2 s$ $2 p$ Lamb shift. The calculation reveals that a reduction of about $0.2 \%$ of the total $2 s-2 p$ Lamb shift is already due to the $2^{+}$-rotational excitation.

In summary we obtained for ${ }_{82}^{208} \mathrm{~Pb} \Delta E\left(1 s_{1 / 2}\right)=101.3$ $\mathrm{meV}, \Delta E\left(2 s_{1 / 2}\right)=17.4 \mathrm{meV}$, and $\Delta E\left(2 p_{1 / 2}\right)=1.4$ meV. Similarly we computed for ${ }_{92}^{238} \mathrm{U} \Delta E\left(1 s_{1 / 2}\right)=$ $1079.2 \mathrm{meV}, \Delta E\left(2 s_{1 / 2}\right)=202.7 \mathrm{meV}$, and $\Delta E\left(2 p_{1 / 2}\right)=$ $23.4 \mathrm{meV}$. Please note that the present numbers deviate slightly from those published in Ref. 13. The level shift for the $2 p_{3 / 2}$ state is completely negligible. The uncertainty in the quoted energy shifts caused by the nuclear polarization is more difficult to estimate compared with radiative corrections in quantum electrodynamics. The numerical error in our computational treatment which is assumed to be below the $1 \%$ level is of minor importance compared with the uncertainty in nuclear parameters, e.g., the nuclear transition strengths. Only the dominant nuclear levels have been taken into account in our evaluations while the majority of states was neglected. Furthermore, we disregarded the spreading width of giant resonances. In average we assign a typical error of $25 \%$ to the final energy corrections for the considered electron bound states.

\section{Estimate for nuclear-polarization effects in superheavy quasiatoms}

Heavy-ion collisions provide the opportunity to study quantum electrodynamical effects in extremely intense electromagnetic fields. During such collisions quasiatomic states are formed where an electron experiences the strong external field of the united charge $Z=Z_{P}+Z_{T}$ of projectile and target nucleus. ${ }^{27,28}$ In collisions slightly above the Coulomb barrier both nuclei are supposed to form a short-living ( $\left.\sim 10^{-21} \mathrm{sec}\right)$ compound system. We now estimate the order of magnitude of nuclearpolarization corrections to the strongly bound quasiatomic $1 s$ state by means of a simplified static model calculation for a system with united charge $Z=170$. We note, however, that the following calculation based on the perturbative treatment as discussed earlier may be an unrealistic description of the situation for several reasons. A first concern may be that the perturbative treatment is no longer adequate in external fields where the coupling constant $Z \alpha>1$. The strongly bound electron is localized near the nuclei so that one cannot neglect possible distortions of the intrinsic nuclear dynamics. A second concern lies in the absence of detailed knowledge about the internal degrees of freedom of short-lived nuclear systems and that the static description has its limitations. Despite these uncertainties we assume that the following calculation provides a rough estimate of the possible magnitude of nuclear-polarization effects in superheavy quasiatoms.

To proceed let us assume that the collective excitations of the superheavy composite system may effectively be described by monopole or quadrupole shape oscillations. We assume an equilibrium radius $R_{0}$ of about $10 \mathrm{fm}$. Instead of deriving a more sophisticated expression for the effective propagator in the framework of the dynamical collective model proposed by Hess and Greiner, ${ }^{29}$ we will use the effective propagators given in Eqs. (27) and (45), respectively.

Calculating first the radial matrix elements one finds a drastic increase of about a factor $10^{3}$ for matrix elements such as $\left|\left\langle 1 s\left|F_{L}\right| E \kappa_{1}\right\rangle\right|^{2}$. The reason for this is the fact that the $1 s$ state is now strongly localized near the nuclei with the large nuclear charge number $Z$. Otherwise one recovers almost the same energy dependence as in the case of heavy atoms discussed previously (see Fig. 4). Having performed the integration over continuum energy the resulting quantities $\tilde{\mathcal{M}}_{1 s_{1 / 2} \mid \kappa_{1}}^{L}$ can be treated as a function of the unknown excitation energies. The time scale involved in the problem suggests possible excitation energies larger than $1 \mathrm{MeV}$. However, considering the $\tilde{\mathcal{M}}_{1 s_{1 / 2} \mid \kappa_{1}}^{L}$ as a function of $E_{L}$ they turn out to be only slowly varying functions of the excitation energy (see Fig. 6). Thus it seems reasonable to introduce an average value $\tilde{\mathcal{M}}_{1 s_{1 / 2} \mid \kappa_{1}}^{L}\left(\bar{E}_{L}\right)$ to eliminate this weak dependence on the unknown excitation energies. The remaining dependence of the energy shift is governed by the reduced electric transition probabilities of this composite system. Figure 7 shows the result for the energy shift due to monopole and quadrupole excitations as a function of the unknown $B(E L)$ values.

Also in this case the monopole vibrations seem to dominate. Our calculation yields a total possible energy shift of the quasiatomic $1 s$ state of the order of about $1 \mathrm{keV}$. We conclude that the major reason for this increase of the effect is due to the increase of the radial matrix elements. Our result based on the perturbative approach does not indicate any significant modification of the value for the critical charge $Z_{\text {cr }}$ at which the $1 s$ states exceed twice the electron rest mass. 


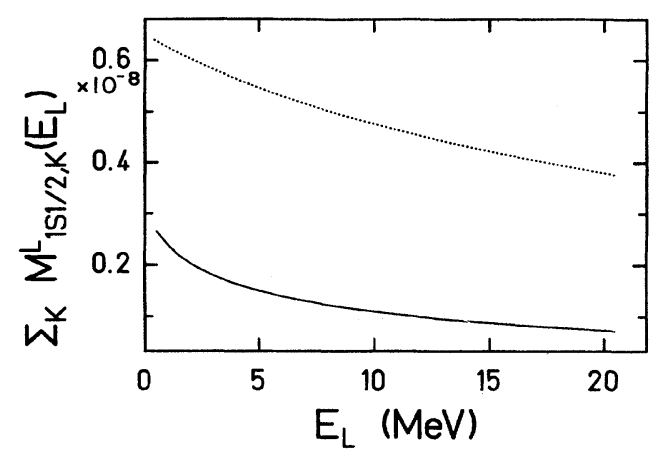

FIG. 6. Energy dependence of $\sum_{\kappa_{1}} \tilde{\mathcal{M}}_{1 s_{1 / 2} \mid \kappa_{1}}^{L}$ as a function of the excitation energy $E_{L}$ for $L=0$ (solid line) and $L=2$ (dashed line).

\section{SUMMARY}

We have presented a systematic approach to the treatment of the effects of nuclear polarization in analogy to the usual QED radiative corrections. Inspired by the notion that a nucleus acts like any other polarizable medium we have utilized the concept of an effective photon propagator with nuclear-polarization insertions. Explicit expressions for the modification of the photon propagator have been derived in the case of virtual collective nuclear excitations like surface vibrations, rotations, and giant resonances. The structure of the effective propagator turns out to be fairly model independent in the sense that it depends only on observables like excitation energies and the corresponding reduced electric transition probabilities.

Based on the Tomonaga-Schwinger equation we have deduced the energy shift of atomic levels. Performing a perturbation expansion one obtains the correction due to nuclear polarization as part of the effective radiative corrections. To lowest order one finds an effective selfenergy and a vacuum polarization diagram containing effective photon lines. When evaluating the self-energy one recovers the terms usually evaluated in the spirit of Schrödinger perturbation theory. Besides these terms we derive an additional vacuum contribution as a consequence of our field theoretical approach which is absent in calculations performed earlier. The nuclear-polarization contribution arising from an analogous vacuum polarization diagram has not been calculated. However, in the case of a nucleus which is characterized by a spherical ground-state charge distribution the induced vacuum polarization charge density is, to lowest order, also spherical symmetric. Thus it can only couple to intrinsic nuclear monopole excitations, while the interaction with higher collective multipole excitations vanishes. Here, we con-

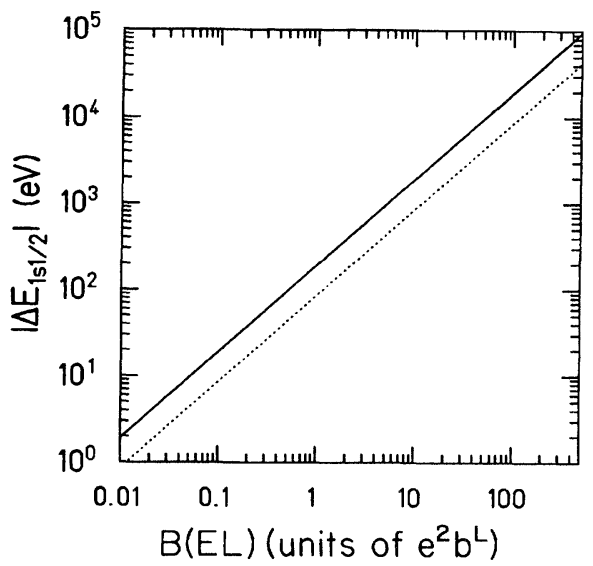

FIG. 7. Contribution of nuclear polarization to the energy shift of the quasiatomic $1 s_{1 / 2}$ state for $Z=170$ as a function of the unknown $B(E L)$ values $(L=0$, solid line; $L=2$, dotted line).

sidered exclusively the effective self-energy.

In particular, we were interested in its contribution to the Lamb shift of K-shell electrons. We found a fair agreement with results obtained in Ref. 7 for ${ }_{82}^{208} \mathrm{~Pb}$. As a second application we calculated the energy shift in ${ }_{92}^{238} \mathrm{U}$ in order to investigate the effect of low-lying rotational states. On the basis of our results we conclude that in uranium polarization effects caused by these collective modes will not be negligible in experiments with extremely high precision. Each nuclear excitation contributes additively to the total energy shift. One may expect a total energy shift of about $0.1 \mathrm{eV}$ in ${ }_{82}^{208} \mathrm{~Pb}$ while nuclear-polarization effects in ${ }_{92}^{238} \mathrm{U}$ yield a correction of about $1 \mathrm{eV}$. The latter should be compared with the total $1 s$-Lamb shift of $458 \mathrm{eV}$ in uranium. The effect of nuclear polarization also decreases the $2 s-2 p$ Lamb shift by about $0.2 \%$ in uranium. The fact that uncertainties due to nuclear polarization enter at the $0.2 \%$ level may indicate that they become relevant in future Lamb-shift experiments aiming at utmost precision tests of quantum electrodynamics.

The scaling of nuclear-polarization effects when calculated perturbatively does not seem to change significantly even in the case of extremely intense fields in superheavy quasiatoms. Our rough estimate of its contribution to the $1 s$-binding energy confirms this. However, we should be aware of the nonperturbative character of nuclearpolarization effects in strong fields which requires in principle a more sophisticated iterative solution of the Dyson equation. The exact knowledge of nuclear-polarization effects would be valuable for the analysis of striking measurements which may further deepen our understanding of QED in strong Coulomb fields.
*Permanent address: Department of Physics, Duke University, Durham, NC 27706.

${ }^{1}$ P. J. Mohr, At. Data Nucl. Data Tables 29, 453 (1983).

${ }^{2}$ W. R. Johnson and G. Soff, At. Data Nucl. Data Tables
33, 405 (1985).

${ }^{3}$ G. Soff and P. J. Mohr, Phys. Rev. A 38, 5066 (1988).

${ }^{4}$ W. Greiner, Z. Phys. 164, 374 (1961).

${ }^{5}$ W. Pieper and W. Greiner, Nucl. Phys. A109, 539 (1968). 
${ }^{6}$ E. Borie and G. A. Rinker, Rev. Mod. Phys. 54, 67 (1982).

${ }^{7}$ B. Hoffmann, G. Baur, and J. Speth, Z. Phys. A 315, 57 (1984).

${ }^{8}$ B. Hoffmann, G. Baur, and J. Speth, Z. Phys. A 320, 259 (1985).

${ }^{9}$ W. Pieper and W. Greiner, Z. Phys. 218, 327 (1969).

${ }^{10}$ B. Müller, J. Rafelski, and W. Greiner, Nuovo Cimento A 18, 551 (1973).

${ }^{11}$ M. Gyulassy, Nucl. Phys. A244, 497 (1975).

${ }^{12}$ G. Soff, P. Schlüter, B. Müller, and W. Greiner, Phys. Rev. Lett. 48, 1465 (1982).

${ }^{13}$ G. Plunien, B. Müller, W. Greiner, and G. Soff, Phys Rev. A 39, 5428 (1989).

${ }^{14}$ G. Plunien, Gesellschaft für Schwerionenforschung Report No. GSI-89-17, ISSN 0171-4546, 1989.

${ }^{15}$ W. H. Furry, Phys. Rev. 81, 115 (1951).

${ }^{16}$ M. Gell-Mann and F. Low, Phys. Rev. 84, 350 (1951).

${ }^{17}$ S. S. Schweber, An Introduction to Relativistic Quantum Field Theory (Harper \& Row, New York, 1961).

${ }^{18}$ G. C. Wick, Phys. Rev. 80, 268 (1950).

${ }^{19}$ J. M. Eisenberg and W. Greiner, Nuclear Models (NorthHolland, Amsterdam, 1987).
${ }^{20}$ A. Bohr and B. R. Mottelson, Struktur der Atomkerne (Akademie-Verlag, Berlin, 1980).

${ }^{21} \mathrm{E}$. M. Rose, Relativistische Elektronentheorie (BI, Mannheim, 1971).

${ }^{22}$ A. I. Achieser and W. B. Berestezki, Quantenelektrodynamik (Harry Deutsch, Frankfurt, 1962).

${ }^{23}$ A. M. R. Joye, A. M. Baxter, M. P. Fewell, D. C. Kean, and R. H. Spear, Phys. Rev. Lett 38, 807 (1977).

${ }^{24}$ J. Barrette, N. Alamanos, F. Auger, B. Fernandez, A. Gillibert, D. J. Horen, J. R. Been, F. E. Bertrand, R. L. Auble, B. L. Burks, J. Gomez del Campo, M. L. Halbert, R. O. Sayer, W. Mittig, Y. Schulz, B. Haas, and J. P. Vivien, Phys. Lett. B 209, 182 (1988).

${ }^{25}$ G. A. Rinker and J. Speth, Nucl. Phys. A306, 360 (1978).

${ }^{26}$ V. P. Varshney, K. K. Gupta, A. K. Chaubey, and D. K. Gupta, Can. J. Phys. 60, 1461 (1982).

${ }^{27}$ W. Greiner, B. Müller, and J. Rafelsk, Quantum Electrodynamic of Strong Fields (Springer-Verlag, Heidelberg, 1985).

${ }^{28}$ W. E. Meyerhof, Science 193, 839 (1976).

${ }^{29} \mathrm{P}$. O. Hess and W. Greiner, Nuovo Cimento A 83, 76 (1984). 